\title{
Pathological changes from the originating to the peripheral sites of Sinonasal Inverted Papilloma are the underlying mechanisms of preoperative MRI-tumor origin prediction*
}

\author{
Siyuan Ma1,"\#, Mu Xian 1,\#, Bentao Yang², Gaoli Fang³, Hongfei Lou'1, Wenling \\ $Y u^{2}$, Xiangdong Wang ${ }^{4,5}$, Junfang Xian'², Xiaohong Song ${ }^{1}$, Erzhong Fan ${ }^{4}$, \\ Ying Li', Luo Zhang ${ }^{1,4,5}$, Chengshuo Wang ${ }^{1}$ \\ Rhinology 58: 1, $59-65,2020$ \\ https://doi.org/10.4193/Rhin19.131 \\ *Received for publication: \\ March 26, 2019 \\ ' Department of Otolaryngology Head and Neck Surgery, Beijing TongRen Hospital, Capital Medical University, Beijing, PR China \\ 2 Department of Radiology, Beijing TongRen Hospital, Capital Medical University, Beijing, PR China \\ Accepted: July 12, 2019 \\ ${ }^{3}$ Department of Otolaryngology Head and Neck Surgery, Beijing DiTan Hospital, Capital Medical University, Beijing, PR China \\ ${ }^{4}$ Beijing Key Laboratory of nasal diseases, Beijing Institute of Otolaryngology, Beijing, PR China \\ \#These authors contributed equally to \\ Department of Allergy, Beijing TongRen Hospital, Capital Medical University, Beijing, PR China \\ this work
}

\begin{abstract}
Background: Our previous study showed that convoluted cerebriform pattern (CCP)-based reverse tracing method in preoperative magnetic resonance imaging (MRI) is a reliable tool in predicting originating site of sinonasal inverted papilloma (SNIP). This study aimed to determine the underlying pathological mechanism of the preoperative MRI-CCP reverse tracing method by assessing the histopathological changes from the origin to the peripheral sites of SNIP.
\end{abstract}

Methodology: The originating site of SNIP was predicted by preoperative MRI in 30 consecutive patients suspected to have primary SNIP. Samples of SNIP originating and peripheral sites were processed by pathological staining for evaluation of stroma score, micro-vessel density (MVD), and tight junction proteins (claudin-5, zonula occludens (ZO)-1 and occludin) expression.

Results: The originating site of SNIP was accurately predicted by preoperative MRI in all patients. Stroma scores, and MVD were significantly greater in the periphery of SNIP than in the originating site. In contrast, Claudin-5 expression in micro-vessels was greater at the originating site than the periphery.

Conclusions: More edematous stroma and intensive micro-vessels with defective tight junction in periphery of SNIP result in more contrast agent diffusing and CCP that can only be observed at the periphery of SNIP on T2 and contrast-enhanced T1 weighted MR images, which may be the mechanisms underlying the CCP reverse tracing method.

Key words: inverted papilloma, magnetic resonance imaging, nose neoplasms, pathology

\section{Introduction}

Sinonasal inverted papilloma (SNIP), as one of the most common benign sinonasal tumors, is characterized by its recurrence tendency, destructive capacity and malignant-transformation possibility ${ }^{(1-4)}$. It is well known that the recurrence is mainly caused by incomplete removal of the SNIP ${ }^{(5)}$, and thus, complete surgical resection, especially the origin of the lesion, is essential for long-term control ${ }^{(3,5-9)}$.

Barns and colleagues ${ }^{(10)}$ noted the gross convoluted surface of
SNIP was like gyri and sulci of the brain; and with a physical examination revealing a polypoid growth covered by a convoluted cerebriform mucosa, the authors first proposed the term convoluted cerebriform to describe the surface mucosa of SNIP. Yousem and colleagues ${ }^{(6)}$ further demonstrated that heterogeneity within the SNIP could be seen in magnetic resonance imaging (MRI) of SNIP as septate striations, which were subsequently described by Ojiri and colleagues ${ }^{(7)}$ as convoluted cerebriform patterns (CCP) of high signal intensity in $\mathrm{T} 2$ weighted images 
(T2WI) and contrast-enhanced T1 weighted images (T1WI). Presently, CCP has become a major defining characteristic of SNIP for radiological diagnosis $(4,8,11-13)$.

Preoperative computed tomography (CT) scans have often been employed to predict original location of SNIP by observing focal hyperostosis of the SNIP's origin ${ }^{(14,15)}$. However, almost $40 \%$ of patients do not show any osteitis signs on $\mathrm{CT}^{(13)}$. Our recent study ${ }^{(13)}$ has demonstrated a high accuracy of predicting the origin of SNIP by CCP-based reverse tracing method in preoperative $\mathrm{MRI}$, as indicated by $95.7 \%$ correct predictions in primary patients. The criteria used for this method were as follows: 1) CCP always occurred just in the periphery but not in the origin of SNIP, 2) On T2WI, the originating site mainly appeared as homogeneous equal signal, and on contrast-enhanced T1WI showed mild enhancement. However, the periphery site often manifested as low-signal-intensity and relatively high-signalintensity striations in T2WI, and contrast-enhanced T1WI well enhancement, and 3) Typically, the SNIP showed a striated septate appearance and spread in a radiating fashion, which allowed tracing the CCP back along the radial texture, to the origin of SNIP.

However, previous studies mainly focused on the clinically diagnostic value of CCP and its underlying pathological mechanism, which was just shown in the periphery of SNIP in most cases. The difference between the origin and the periphery of SNIP, especially the histopathologic features in the originating site, was rarely reported. Thus, the aim of the present study was therefore to assess the histopathological changes from the origin to the peripheral sites of SNIP to determine the mechanism of the preoperative $\mathrm{MRI}-\mathrm{CCP}$ reverse tracing method.

\section{Materials and methods}

\section{Subjects and study design}

This prospective study was approved by the Medical Ethics Committee of Beijing TongRen Hospital and written informed consent was obtained from each participant prior to entering into the study.

The study recruited 30 consecutive patients with primary unilateral neoplasm, who had undergone MRI examination, and found to have CCP in T2WI and contrast-enhanced T1WI. These patients subsequently underwent primary surgery for SNIP removal in Beijing TongRen Hospital, during the period from November 2014 to December 2015. Tissue samples were obtained from the originating site and the periphery of the SNIP in all patients, and processed for histologic and immunohistological evaluation. Patients with concomitant squamous cell cancer and atypical hyperplasia were excluded from the study.

\section{MRI}

All preoperative MRI was performed on a 1.5-T unit (Signa Twin Speed Excite, GE Healthcare, France) or 3-T unit MR (SignaHDx,
GE Health-care, France). Precontrast axial and coronal T1 weighted spin-echo images (TR/TE/NEX, 500-600 ms/ 10-15 ms/2) and $\mathrm{T} 2$ weighted fast spin-echo images (TR/TE/NEX, 3000-3500 ms/120-130 ms/1) were obtained, and immediately after administration of gadopentetate dimeglumine (Gd-DTPA) (Magnevist, Schering, Germany) at a dose of $0.1 \mathrm{mmol} / \mathrm{kg}$ at a rate of $2 \mathrm{ml} /$ sec followed by $20 \mathrm{ml}$ normal saline, contrast- enhanced axial, coronal, and sagittal T1 weighted spin-echo images (with and without fat saturation) were obtained. The same imaging parameters (section thickness of 4-5 mm, intersection gap of $0.5 \mathrm{~mm}$, matrix of $256 \times 256$, bandwidth of $41.67 \mathrm{kHz}$, and FOV of $20 \times 20$ $\mathrm{cm})$ were applied in all the patients enrolled.

\section{Prediction of SNIP originating site}

All MR images were analysed by two radiologists with more than 10 years of experience in head and neck imaging. In order to reduces the bias caused by clinical data (eg. the location of the initial symptom, the location description on the diagnoses, etc), both radiologists were blinded to the medical histories and diagnoses of the patients. In case of disagreement between their assessments of any image, a consensus was reached by reviewing the images within the research team.

Using the method previously described ${ }^{(13)}$, the originating location of the SNIP was predicted from preoperative MRI by CCP-based reverse tracing method, and the actual attachment of the SNIP was confirmed at surgery.

\section{Histologic and immunohistochemistry staining} SNIP specimens collected from the originating site and the periphery of the SNIP of each patient were fixed in $10 \%$ formaldehyde overnight at $4^{\circ} \mathrm{C}$ and processed further according to standard procedures for hematoxylin and eosin (H\&E) staining and immunohistochemical staining for CD34, claudin-5, zonula occludens (ZO)-1 and occludin (antibodies all purchased from Thermo Fisher Scientific, USA). Negative controls were prepared by incubating duplicate sections in the absence of primary antibodies.

Histologic and immunohistochemical assessment The H\&E stained sections were evaluated for the presence of stroma, by examination of 10 consecutive fields at $100 \times$ magnification scored on a scale of 1-4: with $1=<25 \%$ stroma, $2=26 \%$ $50 \%$ stroma, $3=51 \%-75 \%$ stroma, and $4=>75 \%$ stroma. The immunohistologically stained sections were evaluated for the density of CD34+ micro-vessels and the expression of claudin-5 in micro-vessels at the originating and the peripheral sites of the SNIP. Micro-vessels were defined as those with a diameter $<20 \mu \mathrm{m}^{(16,17)}$, and based on the microvascular count standard by Weidner et al. ${ }^{(18)}$, the density of CD34+ micro-vessels was evaluated in 10 consecutive fields at $\times 100$ magnification. According to the method used for SNIPs ${ }^{(19,20)}$, the expression of claudin-5 

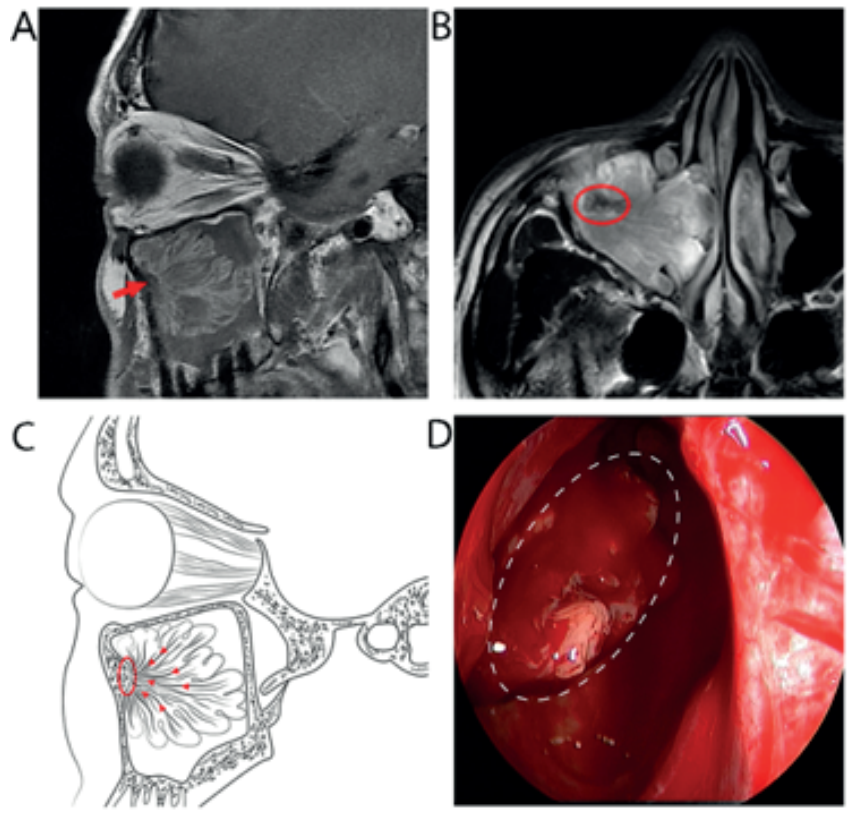

Figure 1. The patient suspected of sinonasal inverted papilloma (SNIP) underwent the modified endoscopic maxillary medial sinusotomy. In magnetic resonance imaging (MRI), contrast-enhanced fat-suppressed T1 weighted images (T1WI) (A) and T2 weighted images (T2WI) (B) expressed characteristic convoluted cerebriform pattern (CCP). The red arrow and circles pointed the originating site of the SNIP. As shown in the schematic diagram of the CCP-based reverse tracing MRI method (C), SNIP grows from the originating site to the periphery. The originating site of the SNIP was easily found on the anterolateral wall of left maxillary sinus (red circle) by tracing back along the growing direction (arrow direction) of CCP. The actual originating site of SNIP was confirmed by surgery ( $D$, inside the white dotted circle).

in micro-vessels was evaluated as immunohistochemical score (IHS), which was calculated by multiplying the percentage of positive micro-vessels and the staining intensity. Staining intensity was subdivided into four categories: $0=$ negative, $1=$ weakly positive, 2 = moderately positive, 3 = strongly positive; and percentage of positive micro-vessels represented the percentage of micro-vessels of each staining intensity.

All samples were evaluated by two independent pathologists with more than 15 years of experience in sinonasal tumors.

Evaluators were blinded to the clinical data of the patients, and the result for each sample was presented as the average value of 10 consecutive fields. In case of disagreement, when the two observers' counts differed by $>10 \%$, a consensus was reached by several members of the research team reviewing the specimen at the same time using a multi-head microscope.

\section{Statistical analysis}

All statistical analyses were carried out by SPSS version 21.0 (IBM Corp., USA). The diagnostic accuracy of CCP to predict SNIP
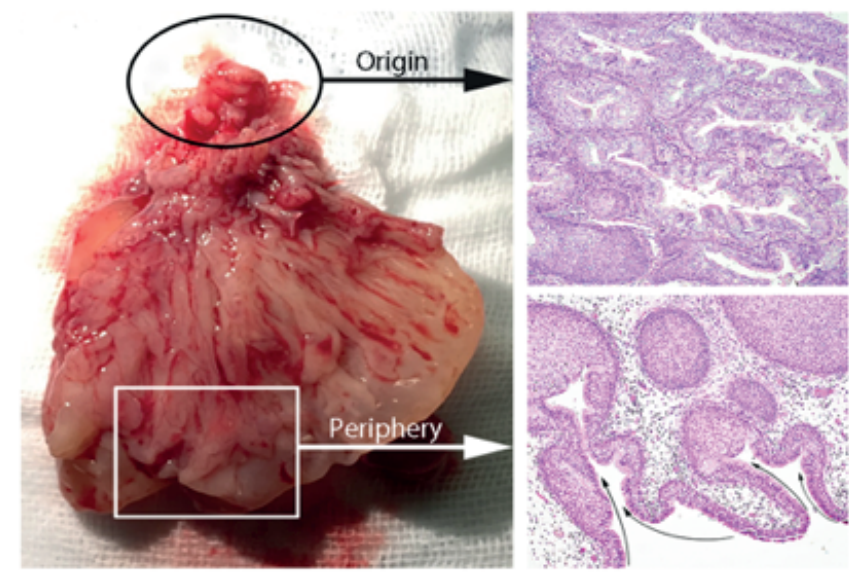

Figure 2. Histological features of SNIP. The gross convoluted surface like gyri and sulci of the brain. Tracing these striated septations back (white rectangle), there is the origin of the SNIP (black circle). The microscopic feature presents as the increasing thickness and hyperplastic epithelium inverting into the stroma (the curve black arrow) from the periphery to the origin ( $\times 100$ magnification).

originating site was evaluated. The data obtained from the histopathologically evaluated samples were expressed as mean \pm SD, and differences between the SNIP origin and peripheral data sets were analyzed using the Wilcoxon matched pairs signed rank test. A value of $\mathrm{p}<0.05$ was considered significant.

\section{Results}

Patient demographics and actual originating site of SNIP The study cohort included 21 males and 9 females, with a mean age of 46.5 years (age range 34-62 years). The most common site of SNIP origin in primary SNIP was the maxillary sinus (36.7\%). SNIPs also originated less frequently from ostiomeatal complex (OMC) (23.3\%), the posterior ethmoid sinus (10.0\%), frontal sinus (10.0\%), middle turbinate (10.0\%), and anterior ethmoid sinus (6.7\%).

\section{Accuracy of originating site prediction by CCP}

Preoperative MR images and findings following surgery (following procedures shown in Figure 1) were compared in all 30 patients to validate the CCP-based reverse tracing predictive method. CCP occurred in all patients enrolled, the median SNIP size was $4.2 \mathrm{~cm}$ in diameter (range, $2.1-7.0 \mathrm{~cm}$ ). Two radiologists had very good agreement on the evaluation of the originated location of SNIP (Kappa=0.952), and the diagnostic accuracy of CCP to predict SNIP originating site was $100 \%$, as confirmed by surgery.

Histopathologic differences between the originating site and the periphery of the SNIP

The gross tumor structure and typical histopathologic mani- 

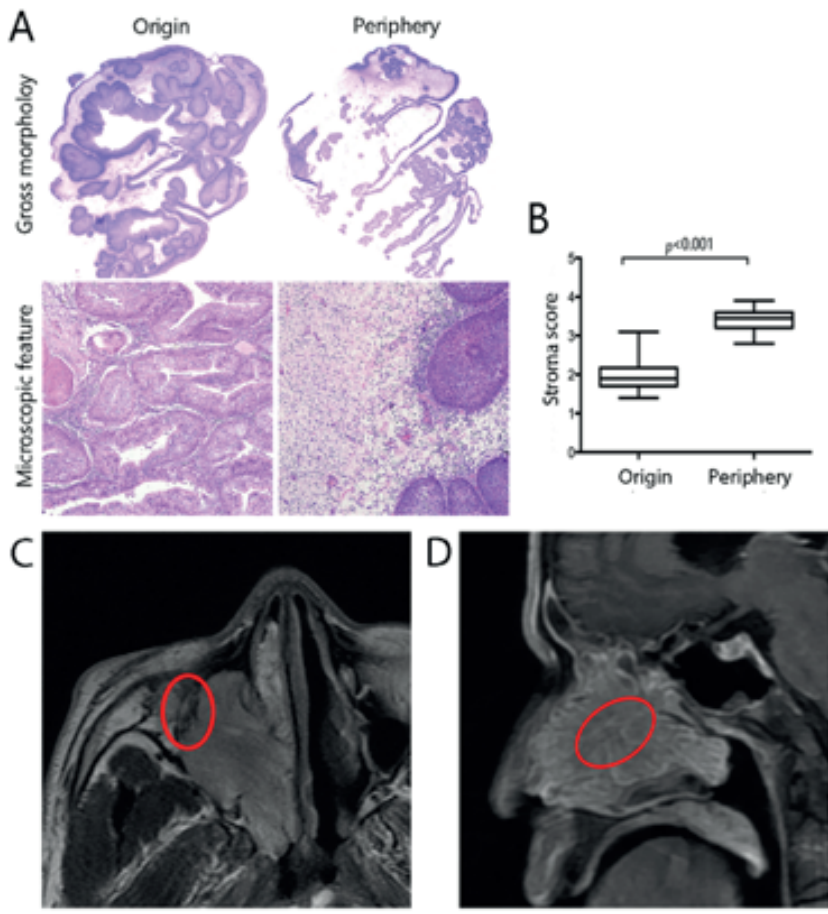

Figure 3. Histopathologic differences between the origin and the periphery of the SNIP. Gross morphology in the originating site $(\times 30$ magnification) and periphery ( $\times 7.5$ magnification) of the SNIP under stereomicroscope. Stroma proportion was evaluated by H\&E staining in the originating site with plaque-like hyperostosis and the periphery of SNIP at $100 \times$ magnification (A). Stroma increased significantly in the periphery of SNIP than in the originating site $(p<0.001)(B)$, which indicated larger interstitial space in the periphery. The difference of stroma proportion and interstitial space in the origin and the periphery lead to the CCP manifestation in T2WI and contrast-enhanced T1WI (C\&D).

festations are shown in Figure 2. The original tissue was more homogeneous and denser than the periphery of the SNIP. At the site of origin, the vast majority of tissue comprised meta-plastic epithelial cells. Stroma increased gradually from the origin to the periphery of the SNIP and varied from being fibrous to edematous. There is a more clustered epithelium, with little or no stroma under the epithelium in the originating site of the SNIP, compared with less inverted epithelium and looser stroma in the periphery of the SNIP (Figure 3A). The mucoserous glands were sparse and the inflammatory components were usually variable and minimal. The stroma score was significantly higher for the periphery than for the originating site of the SNIP (Figure 3B; Table 1), and it also indicated for more interstitial space in the periphery of SNIP. These factors resulted in the manifestation of CCP in the periphery of SNIP in T2WI and contrast-enhanced T1WI (Figure 3C \& 3D).

Micro-vessels in the SNIP tissue around the originating site were confined to narrow fibrous connective tissue, and spread along the columnar stroma between clustered SNIP epithelia. Arteries

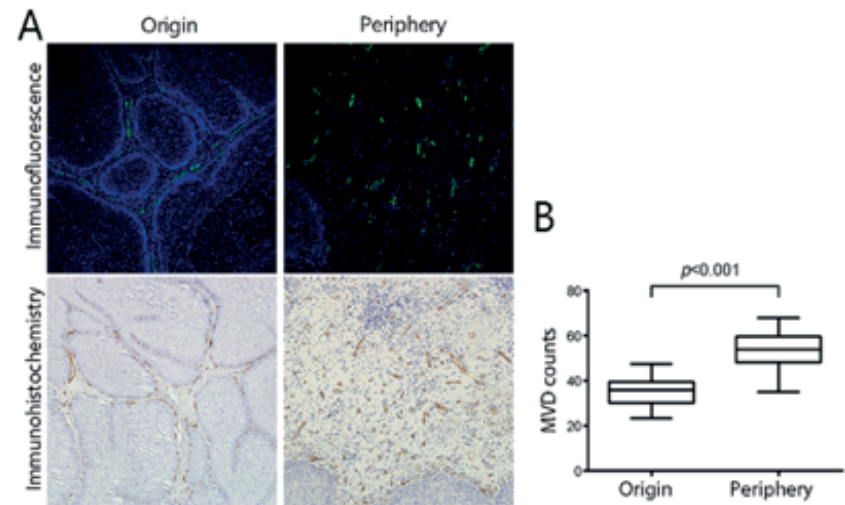

Figure 4. According to the immunofluorescence staining for CD34 in the origin and the periphery of SNIP, more micro-vessels characterized by dilated, tortuous, and hyper-permeable vessels and thin-layer vessel wall were observed in the periphery of the SNIP compared with the origin ( $\times 100$ magnification) (A). Micro-vessel density (MVD) was evaluated by anti-CD34 immunohistochemical staining in the originating site and the periphery of SNIP at $100 \times$ magnification. MVD increased to a significantly greater extent in the periphery of SNIP compared to the originating site $(p<0.001)(B)$. More intensive micro-vessels in the periphery lead to the CCP manifestation in contrast-enhanced T1WI.
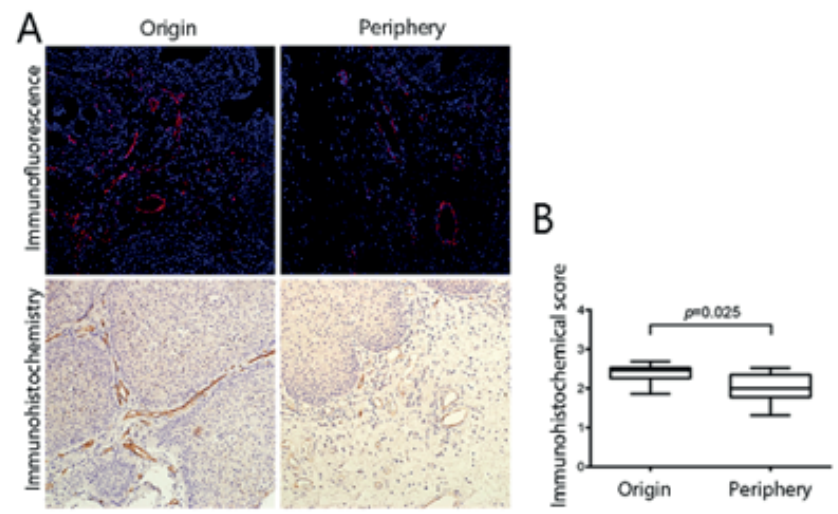

Figure 5. Immunohistochemical staining for claudin-5 in the origin and the periphery of the SNIP. Anti-claudin-5 immunofluorescence and immunohistochemical staining expressed significantly stronger claudin-5 positivity in the origin than the periphery of the SNIP $(\times 200$ magnification) (A). Immunohistochemical score (IHS) was evaluated by immunohistochemical staining in the originating site and the periphery of SNIP at $200 \times$ magnification. The difference of IHS in the periphery and the origin ( $p=0.025)(B)$. Hyper-permeability caused by loss of claudin-5 lead to intense CCP in contrast-enhanced T1WI.

with vascular smooth muscle were mostly present at the originating site of the SNIP. At the periphery of the SNIP, microvessels were characterized by dilated and tortuous vessels, and widely distributed in the loose stroma (Figure 4A). Expression of CD34 was detected in all specimens; with the average MVD determined by CD34 staining being significantly higher in the 
Table 1. Radiologic and histopathologic differences between the origin and periphery of SNIP $(N=30)$.

\begin{tabular}{lllll} 
& Features & Origin & Periphery & \multicolumn{1}{c}{ P value } \\
\hline Radiologic & T2WI & homogeneous equal signal & striations of high signal intensity & - \\
& Contrast-enhanced T1WI & mild enhanced signal & well enhanced signal & $<0.001$ \\
Histopathologic & Stroma score & $1.92 \pm 0.26$ & $3.45 \pm 0.23$ & $52.48 \pm 8.244 / \mathrm{HPF}$ \\
& Micro-vessel density counts & $35.20 \pm 6.008 / \mathrm{HPF}$ & $2.009 \pm 0.402$ & 0.001 \\
& $\begin{array}{l}\text { Immunohistochemical score of } \\
\text { claudin-5 expression }\end{array}$ & $2.386 \pm 0.252$ &
\end{tabular}

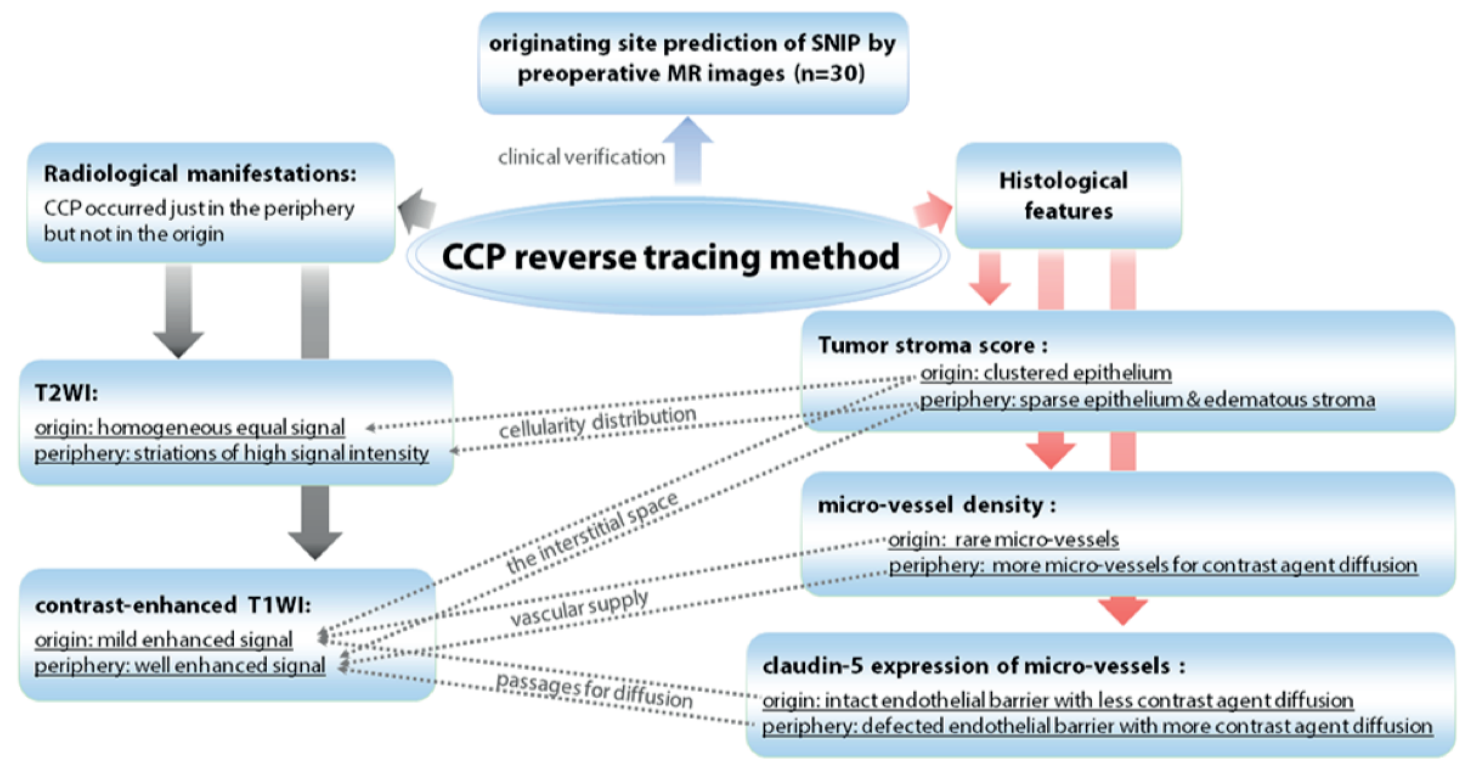

Figure 6. Experimental scheme of the study. Black arrows indicate the different radiological manifestations of originating and peripheral sites of SNIP, and the red arrows indicate the histological features of the originating and peripheral sites of SNIP. Dotted arrows indicate the point to point MRIhistopathologic mechanisms of CCP reverse-tracing method.

periphery than in the originating site of the SNIP (Figure 4B, Table 1), which strengthen the change of contrast-enhanced T1WI in the peripheral site of SNIP.

Furthermore, the micro-vessels in the periphery had thinner walls and lower expression of tight junctions than the microvessels in the originating site (Figure 5A). The defective endothelial layer was hyper-permeable to the contrast agent used for $\mathrm{MRI}$, and resulted in more agent diffusing within the peripheral stroma. Positive expression of claudin- 5 was observed in $80 \%$ of the slides (all in pairs); with the IHS of claudin-5 positive micro-vessels for the originating site of the SNIP being signifcantly higher than for the periphery of the SNIP (Figure 5B, Table 1 ), which reinforce the contrast agent diffusing in contrastenhanced T1WI. The expression of ZO-1 and occludin was poor in micro-vessels, whereas epithelium showed no difference between the origin and the periphery. The schema of all this research is demonstrated in Figure 6.

\section{Discussion}

The present study has partially confirmed the findings for the high accuracy of CCP reverse-tracing method as a means to locate the origin of SNIP, based on different manifestation in MR images between origin and periphery of the SNIP ${ }^{(13)}$. More importantly, this study further explored the underlying histopathological changes, and demonstrated that the origin of SNIP exhibited a dense hyperplastic squamous epithelium inverting into fibrous stroma, while the periphery of the SNIP exhibited a sparse squamous epithelium inverting into the polypoid edematous stroma, which resulted in the classical peripheral CCP on MR images. Indeed, a significantly higher stroma score, indicating more interstitial space in the periphery, and significantly higher MVD and claudin-5, indicating more micro-vessels with more defective endothelial barrier properties in the periphery of SNIP, explains the greater diffusion and presence of contrast-enhanced agent resulting in the CCP in contrast-enhanced T1WI. In contrast, the origin of SNIP presented as homogeneous imaging and light enhancement without any CCP.

In this study, the accuracy of predicting the originating site using this method was found to be $100 \%$; however, this is not entirely unexpected because the patients recruited to the study were primary cases with typical MRI features.

The different distribution of stroma and epithelial in the origin 
and the periphery indicated that in T2WI of SNIP, the lower signal-intensity striations were considered to correspond to the higher cellular metaplastic epithelium and the higher signalintensity striations related to less cellular edematous stroma, which possibly explaining the absence of CCP in the originating site, but not in the periphery of SNIP.

Moreover, the CCP on contrast-enhanced T1WI was clearer than on T2WI. It is a well established fact that lesion enhancement is determined by a variety of factors. However, previous studies indicated that three factors were important for MRI contrast enhancement in any tissue; including an efficient vascular supply, "leaky" intercellular junctions for the contrast agent to effuse out of the vessels, and increased interstitial space to sequester the contrast agent ${ }^{(21-23)}$. In accordance with this, the present study has demonstrated that all the factors facilitating the diffusion of contrast enhancement agent were significantly increased in the periphery of the SNIP compared to the origin of the SNIP. In particular, more dilated and tortuous micro-vessels were distributed in the edematous stroma of the SNIP's periphery, resulting in a significantly greater MVD and consequently a larger microvascular supply of contrast agent for diffusion. In contrast, the SNIP origin had lower MVD, which contributed to low enhancement on contrast-enhanced T1WI.

Secondly, our study revealed a significantly weaker expression of claudin- 5 in the periphery of the SNIP than in the origin, suggesting that the endothelial barrier in the micro-vessels was likely to be weakened and thus provide a route for Gd-DTPA contrast agent, which generally does not cross intact cell membranes ${ }^{(21,24)}$, to diffuse out to a greater extent in the periphery of the SNIP than in the originating site of the SNIP. Claudin-5 is a major component of endothelial tight junction (TJ) strands in non-neuronal tissues, and shown to a reliable marker of vascular endothelium ${ }^{(25-27)}$. Moreover, the endothelial barrier in blood vessels has been shown to tightly regulate the passage of fluids and molecules ${ }^{(28)}$, in which the tight junctions play an important role ${ }^{(29)}$.

Thirdly, our study demonstrated that the periphery site of the SNIP had a larger interstitial space compared to the SNIP originating site. Following injection, the Gd-DTPA contrasting agent diffused rapidly into the extravascular space until it reached the equilibration between extra- and intravascular compartments. In this regard, the origin of SNIP, with a smaller interstitial space, sequestered less contrast-enhanced agent than the periphery of the SNIP; with the different amounts of sequestered Gd-DTPA presenting different appearances on contrast-enhanced T1WI. To our knowledge, no studies has correlated the preoperative MRI-CCP reverse tracing method to detailed histology findings, but in MRI studies of breast tumors, some literatures evaluating the relationship between peripheral rim enhancement and histologic features might be considered discrepant to our findings. Studies by Teifke et al. ${ }^{(30)}$ and Boné et al. ${ }^{(31)}$ showed no relation- ship between MVD and contrast enhancement. The explanation for the difference is probably the fact that the contrast enhancement of SNIP that explain the MRI method is determined by the entire status of vessels (eg. permeability), rather mere number of vessels ${ }^{(23,32)}$.

However, the current study has several limitations. Firstly, as SNIP patients without CCP were not included, this may be of relevance as previous studies have shown the sensitivity of CCP to range from $97.2-100 \%{ }^{(13,33)}$. Thus, SNIP patients without CCP may demonstrate different histopathological changes and therefore need to be investigated more thoroughly in future studies. Similarly, this limitation is equally applicable for the SNIP patients with recurrence and SNIP patients with concomitant malignant transformation that were not included in the current study, and possibly demonstrate pathological features and underlying mechanisms of these SNIP different to those demonstrated in the present cohort of patients. Besides, the limited number of patients in each originating site (eg. maxillary, ethmoids, sphenoid, frontal sinuses, etc) of SNIP made it difficult to demonstrate the radiologic and histopathologic differences between different sites of the origin, which restricted the clinical significance of this study and needs our further efforts.

\section{Conclusion}

In conclusion, CCP-based reverse-tracing method in MRI is a reliable tool in predicting the originating site of SNIP. Our findings further demonstrate that differences in cellularity, vascularity and claudin-5 expression in micro-vessels may be the underlying pathological mechanisms between the originating site and periphery of SNIP. Thus, a better detection of SNIP originating site by preoperative MRI and better understanding of the pathological features of SNIP originating site and periphery may lead to better treatment strategy for management of SNIP originating site, which could further improve surgical plan and reduce recurrence rate.

\section{Acknowledgements}

This study was made possible by grants provided by National Key R\&D Program of China (2016YFC20160905200); national natural science foundation of China $(81630023,81400444$, 81371104, 81470678 and 81420108009); program for Changjiang Scholars and Innovative Research Team (IRT13082); Beijing Municipal Administration of Hospitals Clinical Medicine Development of Special Funding Support (ZYLX201310); Beijing Natural Science Foundation (Z141107002514122); Capital Health Development Foundation (2016-1-2052) and Beijing Talents Project (2016).

\section{Authorship contribution}

$S M$ and MX were contributed equally to complete the experiments, analyse the data and prepare the manuscript; $\mathrm{BY}, \mathrm{GF}, \mathrm{HL}$, 
WY, XW, JX and XS provided input to study design and subject recruitment; $E F$ and $Y L$ were involved in the experiments and data collection; CW and LZ were responsible for the overall design, data analysis and the manuscript revising.

\section{Conflict of interest}

The authors state that they have no conflict of interest.

\section{References}

1. Katori H, Nozawa A, Tsukuda M Histopathological parameters of recurrence and malignant transformation in sinonasal inverted papilloma. Acta Otolaryngol 2006;126:214-218.

2. Elliot A, Marklund L, Hakansson $\mathrm{N}$, et al. Incidence of IP and risk of malignant transformation in the Swedish population 1960-2010. Eur Arch Otorhinolaryngol 2017;274:1445-1448.

3. Vrabec DP. The inverted Schneiderian papilloma: a clinical and pathological study. Laryngoscope 1975;85:186-220.

4. Kasbekar AV, Swords C, Attlmayr B, Kulkarni T, Swift AC. Sinonasal papilloma: what influences the decision to request a magnetic resonance imaging scan? J Laryngol Otol 2018;132:584-590.

5. Busquets JM, Hwang PH. Endoscopic resection of sinonasal inverted papilloma: a meta-analysis. Otolaryngol Head Neck Surg 2006;134:476-482.

6. Yousem DM, Fellows DW, Kennedy DW, Bolger WE, Kashima H, Zinreich SJ. Inverted papilloma: evaluation with MR imaging. Radiology 1992;185:501-505.

7. Ojiri H, Ujita M, Tada S, Fukuda K. Potentially distinctive features of sinonasal inverted papilloma on MR imaging. AJR Am J Roentgenol 2000;175:465-468.

8. Chawla A, Shenoy J, Chokkappan K, Chung R. Imaging Features of Sinonasal Inverted Papilloma: A Pictorial Review. Curr Probl Diagn Radiol 2016:45:347-353.

9. Chiu AG, Jackman AH, Antunes MB Feldman MD, Palmer JN. Radiographic and histologic analysis of the bone underlying inverted papillomas. Laryngoscope 2006;116:1617-1620.

10. Barnes L, Verbin RS, Gnepp DR. Diseases of the nose, paranasal sinuses, and nasopharynx. In: Barnes L, eds. Surgical pathology of the head and neck. New York: Marcel Dekker, 1985; 403-451.

11. Anari S, Carrie S. Sinonasal inverted papilloma: narrative review. J Laryngol Otol 2010;124:705-715.

12. Wang $X$, Zhang Z, Chen $X$, Li J, Xian J. Value of magnetic resonance imaging including dynamic contrast-enhanced magnetic resonance imaging in differentiation between inverted papilloma and malignant tumors in the nasal cavity. Chin Med J (Engl) 2014;127:1696-1701.

13. Fang G, Lou H, Yu W, et al. Prediction of the originating site of sinonasal inverted papilloma by preoperative magnetic resonance imaging and computed tomography. Int Forum Allergy Rhinol 2016;6:1221-1228.
14. Lee DK, Chung SK, Dhong HJ, Kim HY, Kim $\mathrm{HJ}$, Bok KH. Focal hyperostosis on CT of sinonasal inverted papilloma as a predictor of tumor origin. AJNR Am J Neuroradiol 2007;28:618-621.

15. Bhalla RK, Wright ED. Predicting the site of attachment of sinonasal inverted papilloma. Rhinology 2009;47:345-348.

16. Morelli A, Donati A, Ertmer C, et al. Shortterm effects of terlipressin bolus infusion on sublingual microcirculatory blood flow during septic shock. Intensive Care Med 2011;37:963-969.

17. De Backer D, Hollenberg S, Boerma C, et al. How to evaluate the microcirculation: report of a round table conference. Crit Care 2007;11:R101.

18. Weidner N, Semple JP, Welch WR, Folkman J. Tumor angiogenesis and metastasis--correlation in invasive breast carcinoma. N Engl J Med 1991:324:1-8.

19. Soslow RA, Dannenberg AJ, Rush D, et al COX-2 is expressed in human pulmonary, colonic, and mammary tumors. Cancer 2000;89:2637-2645.

20. Remmele W, Schicketanz KH Immunohistochemical determination of estrogen and progesterone receptor content in human breast cancer. Computerassisted image analysis (QIC score) vs. subjective grading (IRS). Pathol Res Pract 1993;189:862-866

21. Ross JS, Delamarter R, Hueftle MG, et al. Gadolinium-DTPA-enhanced MR imaging of the postoperative lumbar spine: time course and mechanism of enhancement. AJR Am J Roentgenol 1989;152:825-834.

22. Han M, Kim TH, Kang DK, Kim KS, Yim H. Prognostic role of MRI enhancement features in patients with breast cancer: value of adjacent vessel sign and increased ipsilateral whole-breast vascularity. AJR Am J Roentgenol 2012;199:921-928.

23. Kuhl CK, Schild HH. Dynamic image interpretation of MRI of the breast. J Magn Reson Imaging 2000;12:965-974.

24. Boehm-Sturm P, Haeckel A, Hauptmann R, Mueller S, Kuhl CK, Schellenberger EA. LowMolecular-Weight Iron Chelates May Be an Alternative to Gadolinium-based Contrast Agents for T1-weighted Contrast-enhanced MR Imaging. Radiology 2018;286:537-546.

25. Soini $Y$. Expression of claudins 1, 2, 3 , 4,5 and 7 in various types of tumours. Histopathology 2005:46:551-560.

26. Morita K, Sasaki H, Furuse M, Tsukita S. Endothelial claudin: claudin-5/TMVCF constitutes tight junction strands in endothelial cells. J Cell Biol 1999;147:185-194.

27. Morita K, Furuse M, Fujimoto K, Tsukita S.
Claudin multigene family encoding fourtransmembrane domain protein components of tight junction strands. Proc Natl Acad Sci U S A 1999;96:511-516.

28. Le Guelte A, Gavard J. Role of endothelial cell-cell junctions in endothelial permeability. Methods Mol Biol 2011;763:265-279.

29. Kluger MS, Clark PR, Tellides G, Gerke V, Pober JS. Claudin-5 controls intercellular barriers of human dermal microvascular but not human umbilical vein endothelial cells. Arterioscler Thromb Vasc Biol 2013;33:489500.

30. Teifke A, Behr O, Schmidt M, et al. Dynamic MR imaging of breast lesions: correlation with microvessel distribution pattern and histologic characteristics of prognosis. Radiology 2006;239:351-360

31. Boné B, Wiberg MK, Parrado C, Falkmer U, Aspelin P, Gad A. Mechanism of contrast enhancement in breast lesions at MR imaging. Acta Radiologica 1998;39:494-500.

32. Matsubayashi R, Matsuo Y, Edakuni G, Satoh T, Tokunaga O, Kudo S. Breast masses with peripheral rim enhancement on dynamic contrast-enhanced MR images: correlation of MR findings with histologic features and expression of growth factors. Radiology 2000;217:841-848

33. Jeon TY, Kim HJ, Chung SK, et al. Sinonasal inverted papilloma: value of convoluted cerebriform pattern on MR imaging. AJNR Am J Neuroradiol 2008;29:1556-1560.

Prof. Chengshuo Wang

No. 1, DongJiaoMinXian Street DongCheng District

Beijing, 100730

China

Tel: +8613911623569

E-mail: wangcs830@126.com

Prof. Luo Zhang

No. 1, DongJiaoMinXian Street

DongCheng District

Beijing, 100730

China

Tel: +86 13910830399

Fax: +86 1085115988

E-mail:dr.luozhang@139.com 